\title{
Design of Slow Wave Structure for G-band TWT for High Data Rate Links
}

\author{
Rupa Basu. Laxma R. Billa, Jeevan M. Rao, Rosa Letizia, Claudio Paoloni \\ Engineering Department \\ Lancaster University \\ Lancaster, UK \\ c.paoloni@lancaster.ac.uk
}

\begin{abstract}
The need of high data rate can be satisfied only by wide frequency bands in the millimetre wave region. This paper presents the design of a G-band $(215-250 \mathrm{GHz})$ Traveling Wave Tube with $40 \mathrm{~dB}$ gain for wireless communications, based on the double corrugated waveguide. The structure of the TWT is based on a single section, instead of the typical configuration of two sections with a sever used at microwave frequency. This is possible due to the high losses at those frequency that permit a stable behaviour. This paper reports both cold and hot simulations.
\end{abstract}

Keywords - TWT, G-band, double corrugated waveguide, millimetre waves

\section{INTRODUCTION}

The G-band (about $205-310 \mathrm{GHz}$ ) offer about $100 \mathrm{GHz}$ useful bandwidth for high data rate internet distribution. So far data rate up to $40 \mathrm{~Gb} / \mathrm{s}$ has been demonstrated [1]. The high atmosphere attenuation and the lack of enough transmission power limit the range to a few tens of meters, even by using high gain antennas. G-band solid-state amplifiers are still in development phase, however, it is unlikely to achieve output power above $50-100 \mathrm{~mW}$. A rough link budget calculation provides that at least one Watt is needed to achieve useful range above $500 \mathrm{~m}$. Recently, traveling wave tubes (TWT) have been considered to as enabling devices for long links at millimetre waves. TWTs have proved to provide multi-Watt output power that satisfy the link specifications [1 - 4].

In this paper, the design ofa G-band $(215-250 \mathrm{GHz})$ TWT, to power a multigigabit per second transmitter, will be described. Simulations of the cold parameters and large signal performance will be described. The TWT is designed with the double corrugated waveguide (DCW) as slow wave structure [5]. Differently, from microwave helix TWTs, it has been designed with a single section without sever. This approach is possible because of the high ohmic losses in the slow wave structure at these frequencies. A single DCW section permits to achieve about $40 \mathrm{~dB}$ gain. In the following, it will be discussed also the stable operation of the TWT.

In a previous work, a DCW with 160 periods [6] was considered for a moderate gain TWT, based on one section without the sever. A study on a longer DCW to achieve about $40 \mathrm{~dB}$ gain is proposed.

\section{TWT DESIGN}

\section{A. Double Corrugated Waveguide Design}

The design of the DCW started with the dimensioning of the geometry of the unit cell to achieve the useful bandwidth with proper beam synchronism, given the beam voltage. The voltage of the electron beam is set at $12.3 \mathrm{kV}$.
This value is a compromise between the best focusing, the power supply cost and the energy of the electrons for a relatively high efficiency. The dimensions were optimised to

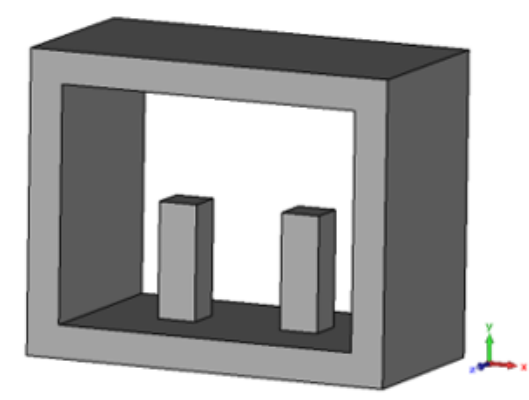

Fig. 1. Double Corrugated Waveguide Unit Cell

assure the proper synchronism of the phase velocity with the electron beam over the $215-250 \mathrm{GHz}$ frequency band.

\section{B. S-parameters}

Once the correct dispersion is achieved, a complete circuit to connect the DCW to the input and output flanges was designed. The output and input coupler consist of a number of pillars tapered in height to provide the $\mathrm{TE}_{10}$ mode at the flanges. The DCW interaction section has 220 periods and each coupler includes 15 periods. The S-parameters of the complete circuit were computed by CST- transient solver. The reflection coefficient $\left(\mathrm{S}_{11}\right)$ better than $-20 \mathrm{~dB}$ is obtained over the desired operating band, assuring a high-quality matching (Fig. 2).

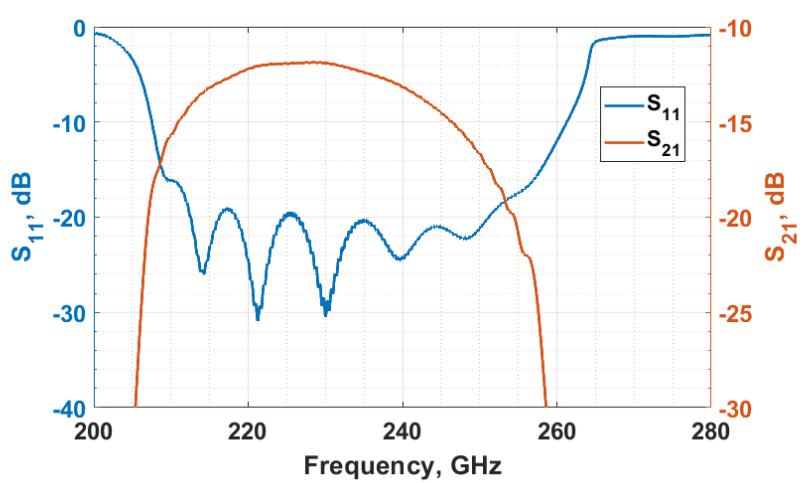

Fig 2. S- parameters for the DCW. 


\section{Large signal analysis}

Particle in cells (PIC) simulations were performed by CST-Particle Studio to compute the large signal behaviour of the TWT. Fig. 3 shows the gain and output power over the frequency band. About $40 \mathrm{~dB}$ gain and more than $8 \mathrm{~W}$ output power, with $1 \mathrm{~mW}$ input signal are obtained over the $215-250$ $\mathrm{GHz}$ band. As further verification, Fig. 4 shows the power as function of time computed at $240 \mathrm{GHz}$, the frequency corresponding to the lower gain value.

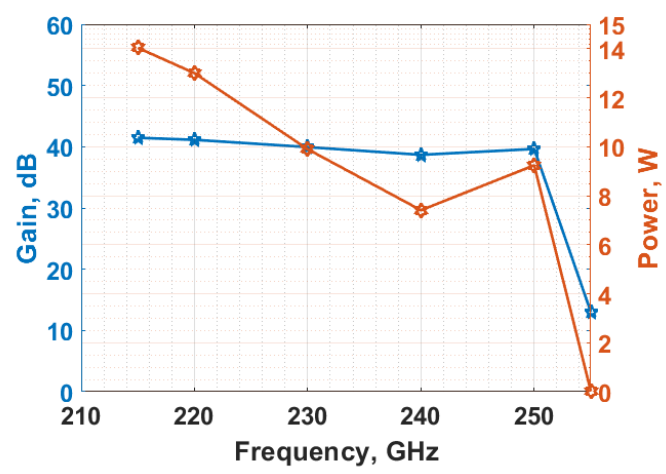

Fig. 3 Gain and power for a total of 220 periods of DCW.

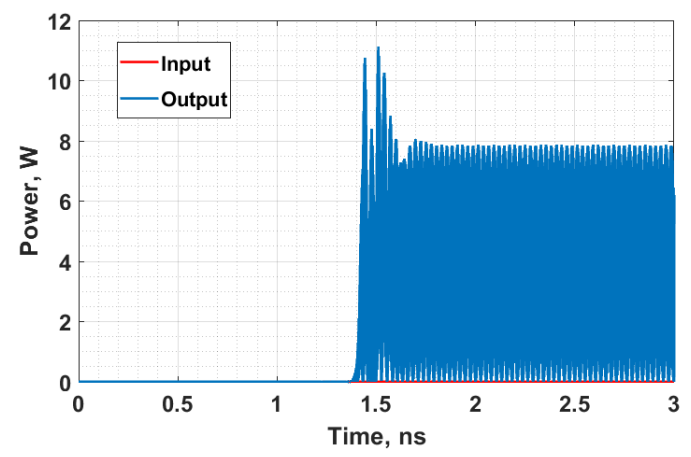

Fig 4. Input and output power signal as function of time computed at $240 \mathrm{GHz}$.

\section{Stability Analysis}

Due to the high gain, a stability analysis was performed to evaluate the risk of oscillations. Typically, a microwave TWT is prone to oscillations if the gain is higher than $20-25 \mathrm{~dB}$ due to the losses are not high enough to supress oscillations.

The condition for stability for a TWT is given by:

$$
\mathrm{Q}=\mathrm{G}-\mathrm{S}_{11}-\mathrm{S}_{22}-\mathrm{S}_{21}<0
$$

where $\mathrm{Q}$ is the stability margin, $\mathrm{G}$ is the gain in $\mathrm{dB}, \mathrm{S}_{11}$ and $S_{22}$ are the reflection losses at the input and output port, and $\mathrm{S}_{21}$ is the circuit loss, in $\mathrm{dB}$.

Figure 5 shows the stability margin as a function of reflection and transmission loss for three gain values $(20,30$ and $40 \mathrm{~dB}$ ). Stability margin values below the stability plane assure a stable operation of the TWT. For the G-Band TWT with 220 periods, the stability is computed, and the points related to the frequencies in the band are plotted in Fig 5. As can be seen, the TWT demonstrates stable operation without the need of sever and attenuators.

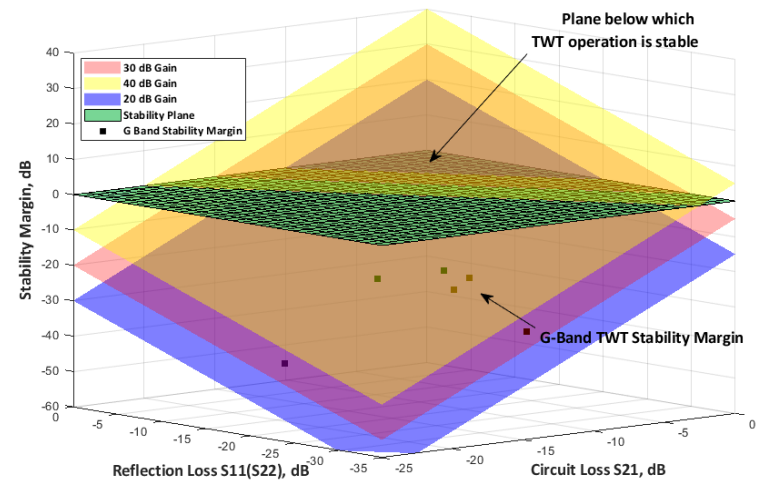

Fig 5 Stability margin and G Band TWT stability

\section{CONCLUSIONS}

The design and performance of a single section G-band TWT with $40 \mathrm{~dB}$ gain in the $215-250 \mathrm{GHz}$ range have been discussed. The double corrugated waveguide was chosen for the G-band TWT due to the simple fabrication and good performance. The single section topology simplifies the fabrication and assembly, very challenging due to the small dimensions related to the wavelength at G-band. The fabrication of the G-band DCW TWT is in progress.

\section{ACKNOWLEDGMENT}

This work has been supported by EPSRC grant EP/P015883/1 in the framework of the CHIST-ERA project TERALINKS, "TERAhertz high power LINKS using photonic devices, tube amplifiers and Smart antennas" and partially supported by ULTRAWAVE project that has received funding from the European Union's Horizon 2020 research and innovation program under grant agreement no 762119.

\section{REFERENCES}

[1] Dhillon, S. S., M. S. Vitiello, E. H. Linfield, A. G. Davies, Matthias C. Hoffmann, John Booske, Claudio Paoloni et al. "The 2017 terahertz science and technology roadmap." Journal of Physics D: Applied Physics 50, no. 4 (2017): 043001.

[2] L. Wenqiang et al., "Development of D-band continuous-wave folded waveguide traveling-wave tube," 2015 IEEE International Vacuum Electronics Conference (IVEC), Beijing, 2015

[3] M. Field et al., "Development of a 100-W 200-GHz High Bandwidth mm-Wave Amplifier," in IEEE Transactions on Electron Devices, vol. 65, no. 6, pp. 2122-2128, June 2018.

[4] Joye, Colin D., Jeffrey P. Calame, Khanh T. Nguyen, and Morag Garven. "Microfabrication of fine electron beam tunnels using UVLIGA and embedded polymer monofilaments for vacuum electron devices." Journal of Micromechanics and Microengineering 22, no. 1 (2011): 015010.

[5] M. Mineo and C. Paoloni, "Double-Corrugated Rectangular Waveguide Slow-Wave Structure for Terahertz Vacuum Devices," IEEE Transactions on Electron Devices, vol. 57, no. 11, pp. 31693175, Nov. 2010.

[6] Basu, Rupa, Laxma R. Billa, Rosa Letizia, and Claudio Paoloni. "Design of sub-THz traveling wave tubes for high data rate long range wireless links." Semiconductor Science and Technology 33, no. 12, $124009,2018$. 